\title{
Les céramiques biomédicales
}

\section{Biomedical ceramics}

\author{
Eric Champion ${ }^{1}$, Amandine Magnaudeix ${ }^{1}$, Patricia Pascaud-Mathieu ${ }^{1}$, Chantal Damia ${ }^{1}$ \\ ${ }^{1}$ Université de Limoges, CNRS, Institut de Recherche sur les Céramiques, UMR 7315
}

RÉSUMÉ. Depuis les civilisations antiques, des céramiques sont utilisées pour réparer des lésions osseuses. Si leur rôle est avant tout fonctionnel, une architecture personnalisée et adaptée au patient entre pleinement dans les critères de fabrication des implants céramiques actuels. Les développements font appel à des procédés innovants de mise en forme associant conception assistée par ordinateur et fabrication additive pour mettre au point des dispositifs complexes destinée à l'ingénierie des tissus osseux. L'Institut de Recherche sur les Céramiques mène à Limoges des travaux de recherche dans ce domaine depuis près de 30 ans et a acquis un savoir-faire basé sur une stratégie dont les principales lignes sont présentées dans cet article.

ABSTRACT. Since ancient civilizations, ceramics have been used to repair bone injuries. Although their role is primarily functional, a personalized architecture adapted to the patient is fully in line with the manufacturing criteria of today's ceramic implants. The current developments implement innovative shaping processes combining computeraided design and additive manufacturing to produce complex devices for bone tissue engineering. The Institute of Research for Ceramics (IRCER) in Limoges has been carrying out researches in this field for nearly 30 years and has acquired a know-how based on a strategy whose main lines are presented in this article.

MOTS-CLÉS. Substituts de greffe osseuse, Céramique, Fabrication additive.

KEYWORDS. Bone graft substitutes, Ceramic, Additive Manufacturing.

\section{La genèse des biocéramiques}

Le squelette est un organe fascinant, à la fois minéralisé et dur mais aussi vivant. Il est constitué chez l'humain adulte de 206 os $^{1}$ aux formes variées et complexes qui assurent de multiples fonctions parmi lesquelles le soutien du corps, la locomotion et la protection des organes vitaux (cerveau, cœur, poumons...). Ils jouent également un rôle clé dans l'alimentation (système maxillaire). De ce fait, l'être humain a de très longue date cherché à réparer les tissus osseux lésés, suites à des traumatique ou des maladies, pour en restaurer les fonctions. Les premiers matériaux exogènes utilisés en tant que xénogreffes sont le fait des civilisations de l'Antiquité et de la Protohistoire. Il s'agissait de dispositifs esthétiques ou fonctionnels visant à réparer des lésions crâniennes, remplacer des dents ou des membres ${ }^{2}$

Depuis l'ère néolithique, la céramique, née de la maîtrise des arts du feu, a constamment accompagné les humains dans leur quête d'amélioration de confort et de qualité de vie. Le domaine médical n'échappe pas à cette relation ancestrale. Ainsi, la première évocation connue de céramique médicale date d'il y a environ 3000 ans et se réfère à une prothèse d'orteil portée par une femme égyptienne ${ }^{3}$. En se limitant à la chirurgie moderne, «l'Art Dentaire» a été l'un des premiers à

\footnotetext{
${ }^{1}$ https://www.lecorpshumain.fr/corpshumain/1-os.html

${ }^{2}$ W. S. Pietrzak, « Musculoskeletal and wound treatment through the ages », dans William S. Pietrzak (dir.), Musculoskeletal Tissue Regeneration. Biological Materials and Methods, Totowa (New Jersey), Humana Press, 2008.

${ }^{3}$ W. A. Wagle, "Toe Prosthesis in an Egyptian Human Mummy », American Journal of Roentgenology, vol. 162, $n^{\circ} 4,1994$, p. $999-1000$
} 
bénéficier de l'usage de céramiques avec, à la fin du XVIIIe siècle, l'implantation des premières couronnes en porcelaine ${ }^{4}$.

\section{Les biocéramiques actuelles}

Aujourd'hui, les céramiques biomédicales, ou biocéramiques, répondent à un large éventail d'applications en tant qu'implants pour la réparation de tissus osseux en chirurgie maxillo-faciale et, plus généralement, en chirurgie orthopédique. De ce fait, elles sont biocompatibles. En d'autres termes, les composés chimiques dont elles sont constituées ne présentent pas de toxicité pour le tissu hôte, ni n'induisent de réaction non désirée et/ou négative. Toutes présentent un point commun : être en contact donc en interaction directe avec les tissus vivants. La nature de ces interactions a conduit à définir deux grandes classes de biocéramiques. La première est dite bio-inerte, apparue dans les années 1970 et qualifiée aujourd'hui de première génération. Elle est principalement constituée de céramiques d'alumine $\left(\mathrm{Al}_{2} \mathrm{O}_{3}\right)$ ou de zircone $\left(\mathrm{ZrO}_{2}\right)$ qui restent chimiquement stable, en simple contact physique sans interaction notable (en cohabitation passive) avec l'organisme hôte même après plusieurs années d'implantation. On retrouve notamment ces céramiques dans les implants dentaires ou dans les prothèses articulaires, les têtes et cupules des prothèses totales de hanche étant l'une des applications les plus connues. Parmi les derniers développements, on peut citer la pose, en première mondiale, d'un sternum en céramique d'alumine par le $\mathrm{D}^{\mathrm{r}}$ Bertin au Centre hospitalier universitaire (CHU) de Limoges en $2015^{5}$.

Une deuxième génération de céramiques est apparue au début des années 1980. Elle est constituée de phosphates de calcium (hydroxyapatite $\mathrm{Ca}_{10}\left(\mathrm{PO}_{4}\right)_{6}(\mathrm{OH})_{2}$ ou phosphate tricalcique $\left.\mathrm{Ca}_{3}\left(\mathrm{PO}_{4}\right)_{2}\right)$ de composition chimique très proche de celle de la partie minérale de l'os humain. Comme les céramiques inertes, elles permettent l'adhérence, la prolifération et la différenciation cellulaire à leur surface, propriété également appelée ostéoconduction. Cette capacité à conduire la repousse osseuse à leur surface est notamment développée par la création d'un réseau de pores dans les pièces (figures 2 et 3 ). Après implantation la porosité est progressivement comblée par des tissus osseux néoformés qui vont coloniser l'intérieur de la céramique. Mais, l'analogie physico-chimique des biocéramiques de seconde génération avec l'os leur confère des propriétés spécifiques. Elles possèdent une excellente biocompatibilité et par opposition aux biomatériaux inertes de première génération qui restent passifs après implantation, n'étant pas dégradés par l'hôte et ne provoquant aucune réponse de l'hôte, elles sont actives. Leur surface peut se dissoudre au contact des fluides biologiques ou de cellules, libérant dans l'environnement biologique voisin des ions calcium et phosphate qui seront réutilisés par l'organisme pour reconstruire de nouveaux tissus osseux à leur contact. Le tissu osseux est en effet en renouvellement permanent dans le corps sous l'action de deux types cellulaires : les ostéoclastes qui ont la capacité de résorber du tissu osseux et les ostéoblastes qui le reconstruisent (figure 1). Après implantation les biocéramiques actives leurrent l'organisme qui ne les considèrent pas comme des éléments exogènes. Les cellules se comportent avec ces céramiques de phosphate de calcium comme s'il s'agissait d'un os naturel, d'où le qualificatif «d'os synthétique » parfois rencontré.

\footnotetext{
${ }^{4}$ https://www.biusante.parisdescartes.fr/aspad/expo91.htm

${ }^{5}$ Lepopulaire.fr, Un an après la première greffe de sternum en céramique. Partie 1 [en ligne], 21 juin 2016 . URL : https://www.youtube.com/watch?v=c7RLOi9otxo (page consultée le 20 novembre 2020).)
} 


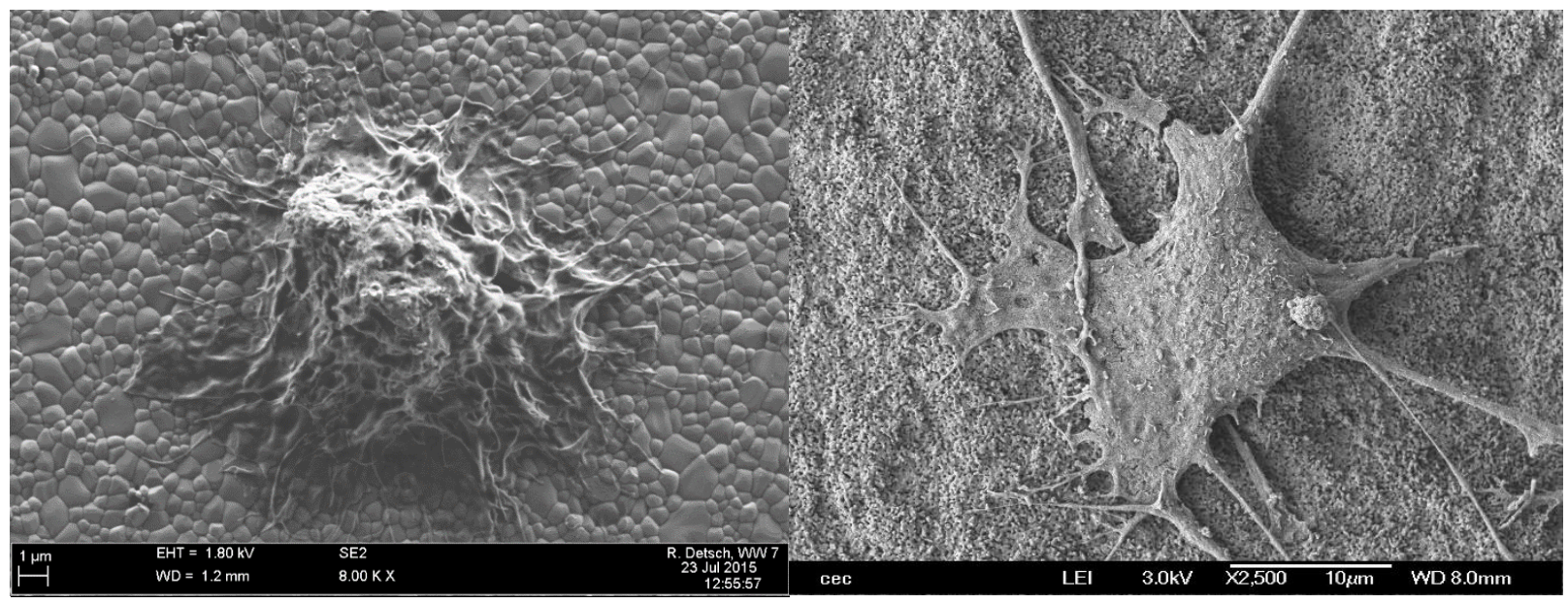

Figure 1. A gauche : Cellule ostéoclaste adhérente à la surface d'une céramique Ces cellules acidifient la surface sur laquelle elles adhèrent pour la dissoudre (crédit photo : Dr Rainer DETSCH, Institut des biomatériaux, Université d'Erlanen-Nuremberg, Allemagne). A droite : Cellules ostéoblastes à la surface d'une céramique. Ces cellules produisent les fibres de collagène sur lesquelles les cristaux d'apatite biologique formant le minéral osseux vont précipiter).

La forte biorésorbabilité ou biodégradabilité de certaines de ces céramiques bioactives, qualifiées de biorésorbables, leur permet, après quelques mois d'implantation, de disparaitre en totalité pour être remplacées par des tissus osseux nouvellement formés. La vitesse à laquelle elles disparaissent de l'organisme est cependant élevée tandis que le processus naturel de formation de nouveaux tissus vivants est lent. De ce fait, seuls de petits volumes osseux peuvent être régénérés ce qui limite l'utilisation de ces céramiques « éphémères ${ }^{6}$ à des implants de petite taille.

Les biocéramiques actives de seconde génération sont couramment implantées sous forme de pièces denses ou poreuses pour le comblement de pertes de substance osseuse. Elles sont également mises en œuvre avec succès en chirurgie maxillo-faciale pour la reconstruction de grandes lésions crâniennes avec la pose, en première mondiale au CHU de Limoges, d'un implant crânien en céramique d'hydroxyapatite phosphocalcique par le Dr Brie en $2005^{7}$. Ces implants céramiques sont fabriqués sur mesure à partir d'outils de CAO/FAO - conception et fabrication assistées par ordinateur - (figure 2). La forme et les dimensions de la pièce sont définies sur une reconstruction en $3 \mathrm{D}$ du squelette du patient, réalisée à partir d'images scanner de ce dernier. L'implant dessiné et simulé en 3D est ensuite mis en forme par une technologie de fabrication additive, la stéréolithographie. Cette technique consiste à consolider une pièce construite couche par couche, d'environ $1 / 10^{\text {ème }}$ de millimètre d'épaisseur, par un procédé d'illumination sélective de la surface d'une couche de pâte photosensible composée d'un mélange de poudre céramique et de monomères photopolymérisables. La pièce ainsi mise en forme est enfin frittée, c'est-à-dire cuite à haute température, typiquement 1200 à $1300^{\circ} \mathrm{C}$ pour les biocéramiques phosphocalciques, pour obtenir au final un implant céramique reproduisant fidèlement l'objet simulé par CAO. Le design personnalisé de ces prothèses aux formes complexes répond à des critères fonctionnels et esthétiques à l'échelle macroscopique. Mais ce design est également personnalisé à l'échelle microscopique. La périphérie de l'implant est « ciselée » avec précision pour obtenir un contact intime avec l'os natif du patient sur tout le pourtour de l'implant lors de sa pose et des pores d'environ un demi-millimètre de section sont présents sur ce pourtour (figure 3). Cette porosité confère à la céramique une architecture interne similaire à celle de l'os spongieux. Après implantation, le volume poreux est

\footnotetext{
${ }^{6}$ « La céramique sert à tout ! » par Stéphanie Belaud, CNRS le journal, n²93, 2018

7 J. Brie et coll., "A new custom made bioceramic implant for the repair of large and complex craniofacial bone defects ", Journal of Cranio-Maxillo-Facial Surgery, vol. 41, 2013, p. 403-407 - https://www.youtube.com/watch?v=10JqDTIxaEw
} 
progressivement colonisé par des tissus vivants conduisant au bout de quelques mois à une parfaite fixation et intégration de l'implant chez le patient. La partie centrale de l'implant est, quant à elle, dense et plus épaisse pour garantir une résistance mécanique élevée sur le long terme et assurer ainsi la protection $\mathrm{du}$ cerveau, fonction première de l'implant, autorisant ainsi la récupération neurologique.
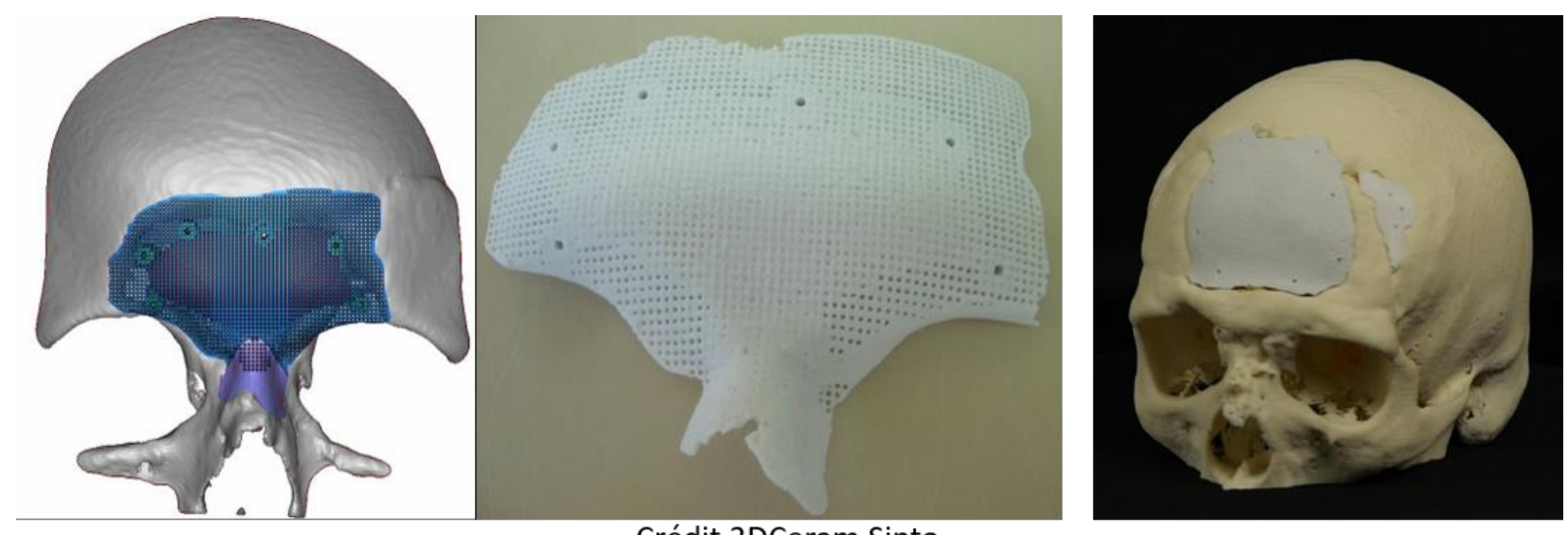

Crédit 3DCeram Sinto

Figure 2. Implants crânio-faciaux en céramique d'hydroxyapatite phosphocalcique mis au point et fabriqués par la société 3DCERAM SINTO en collaboration avec I'IRCER et le CHU de Limoges. À gauche : conception assistée par ordinateur de la pièce sur la visualisation en 3D du crâne du patient construite à partir d'images scanner. Au centre : pièce céramique après frittage. On remarquera que la périphérie de l'implant est poreuse (les six trous de plus grande taille sont destinés à la fixation primaire de l'implant lors de la pose par le chirurgien). À droite : simulation de la pose d'un implant céramique multi-pièces sur une reproduction du crâne du patient (obtenu à partir d'images scanner) fabriqué en résine polymère.

Toutes les céramiques médicales utilisées en chirurgie osseuse sont conçues en premier lieu pour permettre de restaurer la fonction lésée : broyage des aliments, locomotion, protection des organes vitaux, etc. À ce rôle principal vient donc s'ajouter, dans certaines situations, un critère esthétique qui fait partie intégrante du traitement. L'Art dentaire précédemment évoqué en a été une des premières illustrations. On retrouve ainsi dans l'approche médicale moderne la vision empreinte d'esthétisme des prothèses fabriquées par les civilisations antiques.

\section{Les biocéramiques de demain}

A l'heure actuelle des implants céramiques sont fabriquées pour le vivant dans une approche bioinspirée qui vise à «mimer» l'os minéral, tant au niveau de la composition chimique de la céramique que de l'architecture de la pièce. Elles présentent néanmoins des limites d'utilisation. Celles-ci sont principalement liées à leur caractère fragile «cassant» ou à leurs propriétés biologiques encore insuffisantes pour être utilisables dans le traitement de certaines pathologies osseuses ou lésions accidentelles ${ }^{8}$. L'apparition récente des techniques de fabrication additive associée aux outils numériques de CFAO ouvre de nouvelles perspectives de développement pour la conception de systèmes innovants et performants que ne permettent pas les techniques traditionnelles de mise en forme des céramiques 9 .

\footnotetext{
${ }^{8}$ D. Marchat, E. Champion, "Ceramic devices for bone regeneration: mechanical and clinical issues and new perspectives " dans Advances in Ceramic Biomaterials, Elsevier WP Publishing, 2017, Chapitre 8, p. 279-311.

${ }^{9}$ E. Champion, A. Magnaudeix, P. Pascaud-Mathieu, T. Chartier, « Advanced processing techniques for customized ceramic medical devices » dans Advances in Ceramic Biomaterials, Elsevier WP Publishing, 2017, Chapitre 14, p. 433-468.)
} 
Les travaux de recherche scientifique menés à l'Institut de Recherche sur les Céramiques (IRCER) visent à mettre au point de nouvelles céramiques pour un meilleur traitement des patients. Notre objectif est d'améliorer l'ostéointégration des implants céramiques. Il s'agit notamment de développer des dispositifs implantables en vue d'applications en ingénierie des tissus osseux qui vise à régénérer les tissus osseux lésés. La céramique devra servir dans un premier temps de support et de guide pour la formation de nouveaux tissus puis sera amenée à disparaitre progressivement pour laisser idéalement place à une régénération complète du capital osseux du patient. A cette fin, la composition chimique et l'architecture poreuse de la céramique sont étudiées pour définir un design ajusté et personnalisé des implants à la fois à l'échelle des cellules vivantes et à celle des individus.

Ces systèmes ne peuvent être conçus sans une connaissance approfondie de la manière dont le vivant interagit avec le biomatériau. Pour parvenir à cette connaissance des cultures cellulaires sont réalisées sur des objets céramiques modèles. Différents procédés de mise en forme des céramiques sont mis en œuvre pour développer des architectures spécifiques d'intérêt incluant un réseau tridimensionnel de pores (figure 3). Des céramiques poreuses peuvent être élaborées en imprégnant des mousses polymères par une barbotine. Par cette méthode, les parois de la mousse polymère sont enrobées par une fine couche de céramique déposée à leur surface. Un traitement thermique de frittage à haute température est ensuite réalisé. Il permet de bruler la mousse polymère support, ne laissant au final que la céramique sous forme d'une pièce poreuse répliquant l'architecture de la mousse polymère de départ. Cette technique simple de réplique ne permet cependant pas d'élaborer directement des architectures complexes et contrôlées. Or, le comportement des cellules (adhésion, prolifération et différenciation) peut être influencé par la topographie de la surface du matériau avec lequel elles sont en contact. Une architecture poreuse contrôlée et adaptée, à l'échelle des cellules, apparait ainsi nécessaire pour obtenir une intégration biologique optimale de l'implant. Pour répondre à cette problématique des pièces sont fabriquées à partir de pâtes céramiques mises en forme par des procédés de fabrication additive faisant appel à la CFAO. De façon similaire à la stéréolithographie, mise en œuvre avec succès pour fabriquer des implants d'architecture macroscopique sur mesure à l'échelle du patient, la microstéréolithographie est développée à l'IRCER pour fabriquer des pièces comportant des pores de géométrie complexe et contrôlée avec une grande précision (de l'ordre de 20 micromètres) à l'échelle des cellules.

L'ostéoconduction, qui permet aux céramiques poreuses d'être colonisées par des tissus vivants, reste toutefois limitée par la capacité de l'organisme à produire naturellement de nouveaux tissus. Comme mentionné plus haut, la néoformation de tissus osseux est un processus lent et seuls de petits volumes de tissus peuvent être régénérés naturellement ${ }^{8}$. Aucun os néoformé par l'organisme n'est observé in vivo au-delà d'environ un centimètre du bord de l'implant en contact avec l'os natif. L'utilisation de ces céramiques poreuses seules ne peut donc pas se concevoir pour régénérer des tissus dans de grands volumes à combler. Pour lever ce verrou, l'association de molécules organiques d'intérêt (e.g. protéines, facteurs de croissance) à la céramique inorganique pour stimuler et favoriser les processus biologiques de vascularisation et de minéralisation de tissus osseux est également étudiée. La micro-extrusion, qui consiste à étaler couche par couche des filaments de pâte, est notamment mise en œuvre pour construire des échafaudages poreux dans lesquels des cavités sont ménagées pour y inclure des gels chargés en molécules biologiques. Ces architectures sont étudiées afin d'aboutir à un design qui permette après implantation la libération progressive et contrôlée des molécules dans l'environnement biologique local ciblé pour une activité optimale. Il s'agit ici de parvenir à accélérer les processus de régénération osseuse au sein de la céramique pour une invasion tissulaire dans un volume important ce que le processus naturel de formation des tissus ne permet pas. L'objectif est d'ouvrir la voie à la réparation de grands défauts de substance osseuse, qui reste une difficulté actuelle en chirurgie, grâce à l'émergence d'une troisième génération de biomatériaux basée sur des céramiques bioactives poreuses, fonctionnalisées par des molécules biologiques, aux propriétés ostéoinductives. 


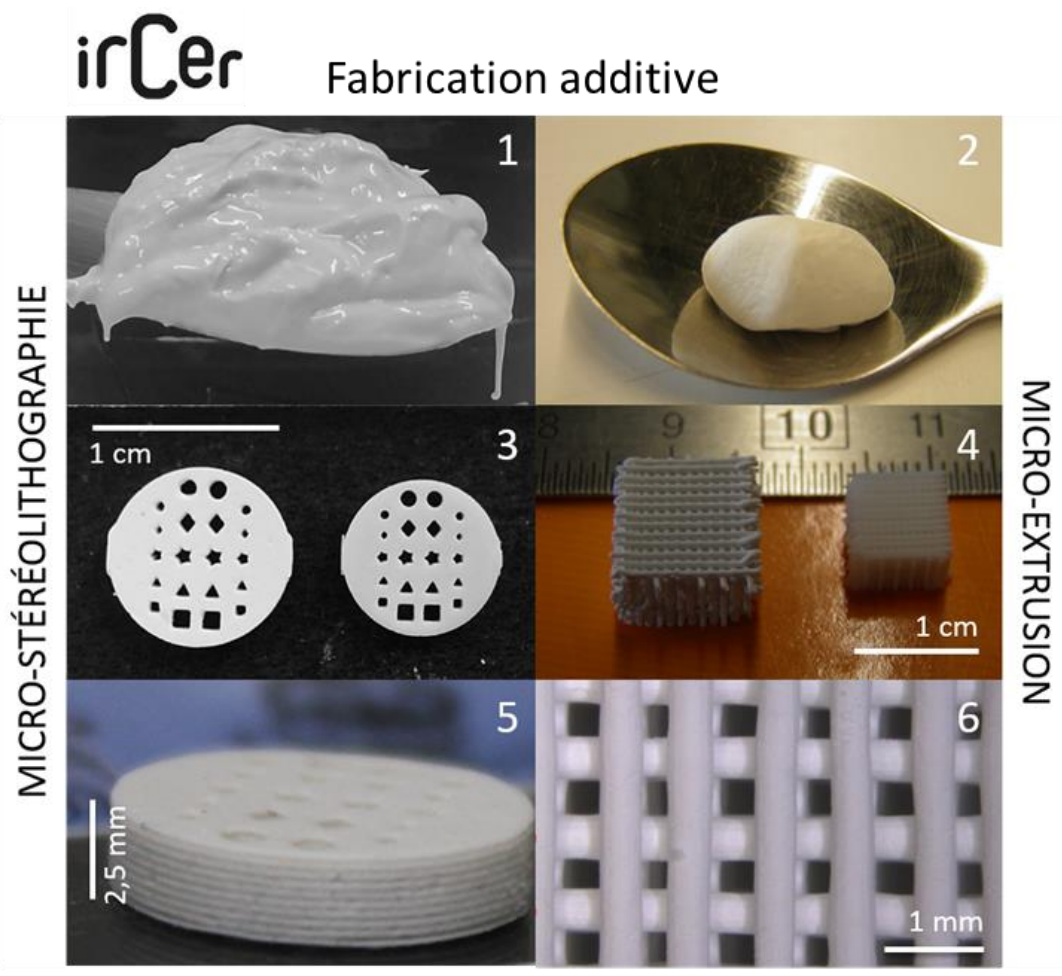

Figure 3. Illustration des procédés de fabrication de biocéramiques poreuses utilisés à l'IRCER. A gauche : suspension céramique et imprégnation d'une mousse de polyuréthane, zoom sur la microstructure poreuse des pièces céramiques. A droite : procédés de fabrication additive ; pâtes céramiques utilisées et exemples de pièces et de réseau de pores obtenus par microstéréolithographie (1,3, et 5) ou micro-extrusion

(2, 4 et 6).

L'évaluation des propriétés et performances biologiques de ces systèmes complexes au cours de leur développement et une condition sine qua non de leur optimisation. Elle est réalisée à l'IRCER par des cultures cellulaires au contact des céramiques. Il s'agit en premier lieu de s'assurer de la biocompatibilité de la céramique en fonction de sa composition chimique. Les éléments qui la constitue et/ou qui peuvent être libérés au cours de sa dégradation en contact avec les liquides biologiques et les cellules, doivent ad minima ne pas engendrer de toxicité vis-à-vis de ces dernières. La capacité des cellules à adhérer et proliférer à la surface de la céramique et dans sa porosité est ensuite évaluée. Enfin, leur aptitude à se différencier pour former de nouveaux vaisseaux sanguins et du tissu osseux à l'intérieur de la porosité et testée en fonction de la composition chimique de la céramique, qu'elle soit fonctionnalisée ou non par des molécules organiques, mais également en fonction de la forme des pores et des courbures de surface de la pièce (figure 4). Ces travaux sur l'influence de la géométrie des pores ont notamment permis de montrer que les cellules sont particulièrement sensibles aux courbures de surface. Les angles aigus (concavité) sont préférentiellement colonisés que ce soit par des cellules osseuses ${ }^{10}$ ou par des vaisseaux sanguins ${ }^{11}$. Comme illustré sur la figure 5 , la vitesse de colonisation des pores de la céramique est influencée par leur géométrie : un pore triangulaire est plus rapidement colonisé qu'un pore circulaire. À leur échelle, c'est-à-dire environ un dixième de millimètre, les cellules sont

\footnotetext{
${ }^{10}$ A. Magnaudeix, J. Usseglio, M. Lasgorceix, F. Lalloué, C. Damia, J. Brie, P. Pascaud-Mathieu, E. Champion, « Quantitative analysis of vascular colonisation and angio-conduction inporous silicon-substituted hydroxyapatite with various pore shapes in a chick chorioallantoic membrane (CAM) model », Acta Biomaterialia, 2016, 38, 179-189.

${ }^{11}$ U. Rudrich, M. Lasgorceix, E. Champion, P. Pascaud-Mathieu, C. Damia, T. Chartier, J. Brie, A. Magnaudeix, Pre-osteoblast cell colonization of porous silicon substituted hydroxyapatite bioceramics: Influence of microporosity and macropore design, Materials Science \& Engineering C, 2019, 97, 510-521.
} 
particulièrement sensibles à la forme de la céramique sur laquelle elles se déplacent et prolifèrent. Il en résulte que des pores de section triangulaire sont beaucoup plus favorables à l'invasion tissulaire et vasculaire que des pores de section circulaire classiquement ménagés dans les implants poreux. Cette connaissance du comportement cellulaire au contact de la céramique permet de définir des critères rationnels pour la fabrication de futurs implants au design optimisé pour des performances biologiques accrues, et donc potentiellement une amélioration de la qualité de soins pour le patient.
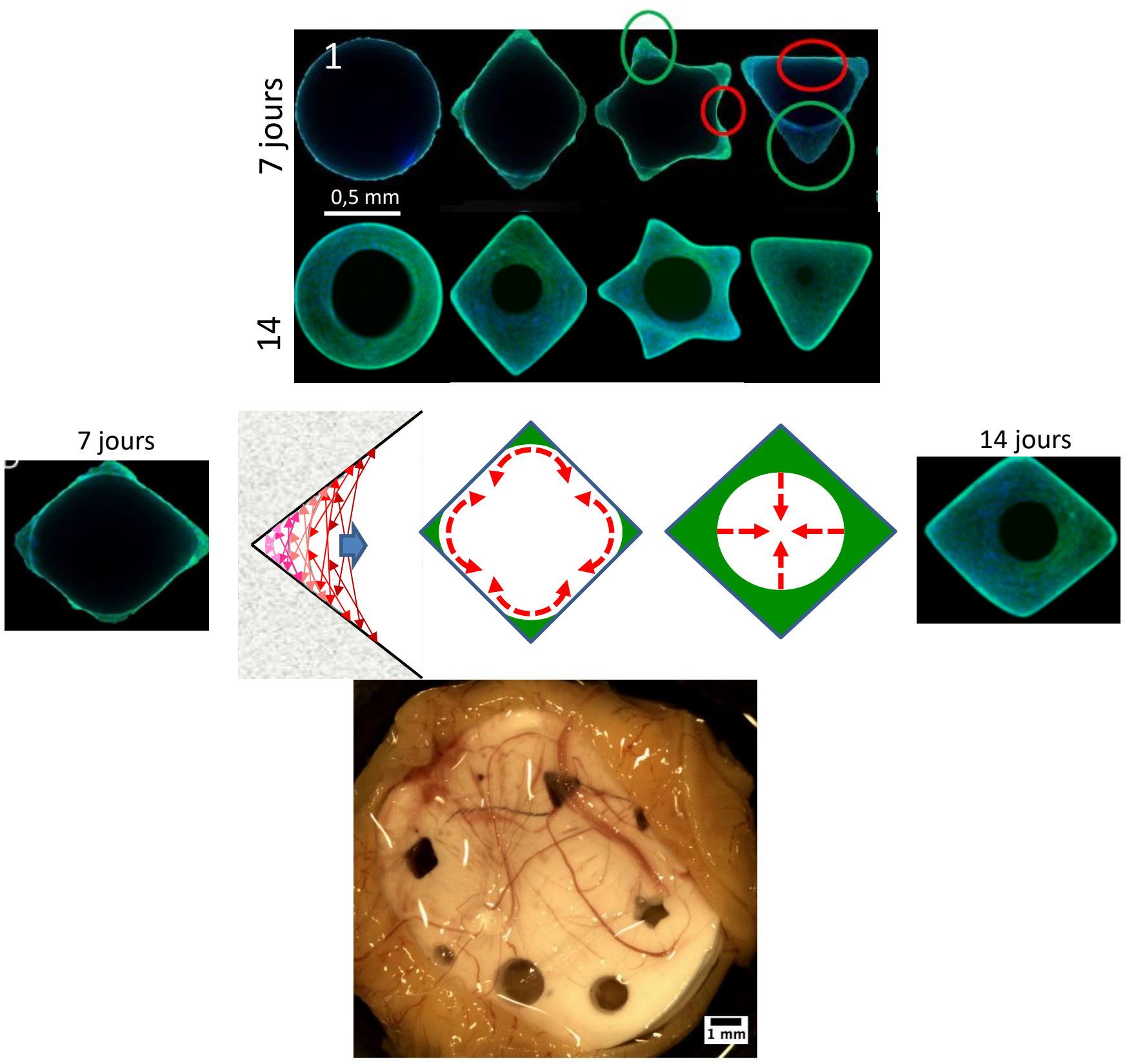

Figure 4. Interactions céramique-vivant. En haut : colonisation cellulaire après 7 et 14 jours de l'intérieur des pores de géométrie variable de la pièce illustrée en figure 3. La coloration fluorescente bleu-vert représente le tapis cellulaire formé : en haut après sept jours de culture (les zones entourées en vert montrent les surfaces peu colonisées et en rouge celles qui sont préférentiellement colonisées). Au milieu :

Schéma de l'organisation cellulaire et de la progression centripète des cellules au cours du temps pour coloniser le pore. Images reproduite de la référence 11, copyright (C 2018, avec l'autorisation d'Elsevier. En bas : Illustration de la colonisation des pores de la céramique par des vaisseaux sanguins, Images reproduite de la référence 10, copyright (C) 2016, avec l'autorisation d'Elsevier.

Les travaux menés à l'IRCER actuellement sur les biocéramiques ont donc pour finalité de passer de biomatériaux de substitution osseuse passifs, ostéoconducteurs, qui supportent la formation naturelle de nouveaux tissus, à des systèmes actifs pour l'ingénierie tissulaire osseuse, capables de 
favoriser la régénération du capital osseux du patient et/ou aptes à délivrer localement des molécules actives, adaptées à des situations pathologiques spécifiques dans le cadre d'une médecine personnalisée. L'ensemble des paramètres chimiques, physiques du matériau et de son environnement biologique sont pris en compte dans une approche scientifique globale. Pour ces nouvelles formes associées au vivant, l'apport de la biologie au génie des procédés céramiques trouve tout son intérêt. 